Commun. Fac. Sci. Univ. Ank. Series $\mathbf{A}_{1}$

V. 39. pp. 1-4 (1990)

\title{
SOME SUBRING PROPERTIES OF THE RING OF HOLOMORPHIC FUNCTIONS ON A NON-EMPTY SUBSET OF AN OPEN RIEMANN SURFACE
}

\author{
NURHAYAT İSPİR I. KAYA ÖZKIN
}

Ankar a University, Faculty of Sciences, Mathematics Department, Tandoğan, 06100 Ankara,

Throughout this paper $R$ and $S$ will denote open Riemann surfaces and $X, Y$ will be non-empty subsets of $R$ and $S$, respectively. A function $\varnothing: X \rightarrow S$ is said to be analytic if for each point $p \in X$ there is an open neighborhood $U_{p}$ of $p$ and an analytic function $\psi_{p}: U_{p} \rightarrow S$ such that $\psi_{p}$ and $\varnothing$ coincide on $U_{p} \cap X$. This is equivalent to assuming that there is a single open set $U \supset X$ and an analytic function $\varnothing: U \rightarrow S$ such that $\psi \mid \mathrm{X}=\varnothing$.Let $\mathrm{A}(\mathrm{X}, \mathrm{Y})$ denote the set of all analytic functions $\varnothing: X \rightarrow S$ with $\varnothing(X) \subset \mathbf{Y}$. For $\mathbf{Y}=\mathbf{S}=\mathbf{C}$, a function in $\mathbf{A}(\mathrm{X}, \mathbf{C})$ is called holomorphic and we write $H(X)=A(X, C)$.

It is well known that $H(X)$ forms a ring under pointwise addition and multiplication. In fact, $H(X)$ is an algebra over both the complex numbers $C$ and the real numbers $I R$.

This paper is concerned with proper subrings $R^{*}$ of $H(X)$ which are isomorphic images of $H(Y)$, the ring of all analytic functions on a non-empty subset $Y$ of an open Riemann surface $S . \Phi$ will denote a homomorphism from $H(Y)$ into $H(X)$ which maps each constant function onto itself; i.e., a C-algebra homomorphism. $\varnothing$ denotes an analytic mapping from $X$ into $Y \subset S$; i.e., $\varnothing \in A(X, Y)$, and $R_{\varnothing}$ the subring of $\mathrm{H}(\mathrm{X})$ which is composed of the functions go $\varnothing$ for $\mathrm{g} \in \mathrm{H}(\mathrm{Y})$. It has been shown that if $\Phi$ is a homomorphism of $H(Y)$ into $H(X)$ and $\Phi$ maps each constant function onto itself, then there is a unique analytic mapping $\varnothing \in \mathrm{A}(\mathrm{X}, \mathrm{Y})$ such that $\Phi(\mathrm{g})=$ go $\varnothing, \mathrm{g} \in \mathrm{H}(\mathrm{Y})[4]$. Thus $\Phi$ $(H, Y))$, the image of $H(Y)$ under $\Phi$, is the subring $R_{\varnothing}$ for some analytic mapping $\varnothing \in \mathbf{A}(\mathrm{X}, \mathrm{Y})$. Now we give some basic definitions and properties of $\mathbf{R}_{\varnothing}$.

A two-dimensional manifold is defined as a connected Hausdorff space $M$ with the property that each point of $M$ is contained in an open set homeomorphic to an open set in the Euclidean plane. The two- 
dimensional manifold $M$ is an analytic manifold or abstract Riemann surface if there is a collection $\left\{\left(U_{i}, \theta_{i}\right): i \in I\right\}$ where for the index set $I$, $\left\{U_{i}: i \in I\right\}$ is an open covering of $M$ and $\theta_{i}$ is a homeomorphism of $U_{i}$ onto an open set in the complex plane. Also, if $U_{i} \cap U_{j}$ is non-empty, then $\theta_{j} \circ \theta_{i}^{-1}$ is a conformal sense-preserving mapping of $\theta_{i}\left(U_{i} \cap U_{j}\right)$ onto $\theta_{j}\left(U_{i} \cap U_{j}\right)$, that is $w=\theta_{j} \circ \theta_{i}^{-1}(z)=f(z)$ is an analytic function of $z$ in $\theta_{i}\left(U_{i} \cap U_{j}\right)$. We say $\left\{\left(U_{j}, \theta_{i}\right): i \in I\right\}$ defines an analytic structure on the manifold $M$, and another collection $\left\{\left(V_{j}, \psi_{j}\right): j \in J\right\}$ defines the same analytic structure if the union of the two sets satisfies the conditions for an analytic structure on M. We say the Riemann surface is open if it is not compact.

If $M$ is a Riemann surface, $(U, \theta)$ belongs to $\left\{\left(U_{i}, \theta_{i}\right): i \in I\right\}$ on $M$, $p_{0}$ belongs to $U$, then $z=\theta(p)$ is a local parameter about $p_{0}$ in $U$ and there is another local parameter $w=\psi(p)$ about $p_{0}$ with $\psi\left(p_{0}\right)=0$ and $|w| \leqq 1$. We define $w=\left(z-z_{0}\right) / r$ where $\theta\left(p_{0}\right)=z_{0}$ and $\left\{z:\left|z-z_{0}\right|\right.$ $\leqq r\}$ is contained in $\theta(U)$. The structure of $M$ is not changed. A complex-valued function $f$ on $M$ is called analytic or holomorphic at the point $p_{0}$ if in terms of the local parameter $z=\theta(p), \theta\left(p_{0}\right)=0$, the function $f\left(\theta^{-1}(z)\right)$ is an analytic function of $z$ for $|z|<r$ for some $r>0$. $f$ is holomorphic on $M$ if $f$ is holomorphic at each point of $M$. If $f$ is a mapping of the Riemann surface $M_{1}$ into the Riemann surface $M_{2}, p_{0} \in M_{1}, f\left(p_{0}\right)$ $=q_{0}, z=\theta(p)$ is a local parameter about $p_{0}, w=\psi(q)$ is a local parameter about $q_{0}$, we say $f$ is analytic on $M_{1}$ if the function $w=\left(f\left(\theta^{-1}\right.\right.$ $(z)))=g(z)$ is an analytic function of $z$ for all $p_{0} \in M_{1}$. The two surfaces $M_{1}$ and $M_{2}$ are conformally equivalent if there is a one to-one analytic mapping of $M_{1}$ onto $M_{2}$ [1], [5].

Suppose $\mathrm{X}$ and $\mathrm{Y}$ are non-empty subsets of open Riemann surfaces $R$ and $S$ respectively. We define a mapping of $H(Y)$ into $H(X)$ by $\Phi(g)=g o \varnothing$ for $g=H(Y)$. go $\varnothing$ is holomorphic on $X$ and $\Phi$ is a homomorphism. The image of $\Phi, R_{\varnothing}=\Phi(H(Y))$ is a subring of $H(X)$. If $\lambda$ is a constant function on $Y$ then $\Phi(\lambda)=\lambda o \varnothing=\lambda$ so $\Phi$ preserves constant functions. C.D. Minda proved that if $R$ and $S$ are open Riemann surfaces and $X, Y$ non-empty subsets of $R, S$ respectively, and if $\Phi$ : $H(Y) \rightarrow H(X)$ is a C-algebra homomorphism, then there is a unique analytic function $\varnothing$ of $X$ into $\mathrm{Y}$ such that $\Phi(\mathrm{g})=$ go $\varnothing$ for $\mathrm{g} \in \mathrm{H}(\mathrm{Y})$ [4]. Also if $\Phi$ is an isomorphism of $H(Y)$ into $H(X)$, then $\varnothing$ is a oneto-one mapping of $X$ into $Y$. Thus a subring $R^{*}$ of $H(X)$ is a homomorphic image of a ring $\mathrm{H}(\mathrm{Y})$ under a $\mathrm{C}$-algebra homomorphism if and only if $\mathrm{R}^{*}=\mathbf{R}_{\varnothing}=\left\{\mathrm{g}_{1} \mathbf{0} \varnothing: \mathrm{g} \in \mathrm{H}(\mathrm{Y}), \varnothing \in \mathrm{A}(\mathrm{X}, \mathrm{Y})\right\} . \mathbf{R}_{\varnothing}$ contains the 
constant functions, denoted by $\mathrm{C}$, since $\mathrm{C} \subset \mathrm{H}(\mathrm{Y})$ and $\Phi(\lambda)=\lambda$ for $\lambda \in \mathbf{C}$.

A relation between $\Phi, \varnothing$, and $\mathbf{R}_{\varnothing}$ is given by the following theorem.

THEOREM 1. Let $R$ and $S$ be open Riemann surfaces and $X$, $Y$ non-empty subsets of $R, S$ respectively. If $\Phi: H(Y) \rightarrow H(X)$ is a ring homomorphism defined by $\Phi(\mathrm{g})=\mathrm{go} \varnothing$ for $\mathrm{g} \in \mathrm{H}(\mathrm{Y}), \varnothing \in \mathrm{A}(\mathrm{X}, \mathrm{Y})$ and if $\mathbf{R}_{\emptyset}=\Phi(\mathrm{H}(\mathrm{Y}))$, then the following three conditions are equivalent:

(a) $\mathbf{R}_{\varnothing}$ properly contains the constant functions,

(b) $\varnothing$ is not a constant function,

(C) $\mathbf{H}(\mathrm{Y})$ is isomorphic to $R_{\varnothing}$.

Proof. Suppose $\mathbf{R}_{\varnothing}$ properly contains $\mathrm{C}$. We shall show that $\varnothing$ is not a constant function. On the contrary, if we suppose that $\varnothing(\mathrm{X})$ $=\{\mathrm{c}\}$, then $\Phi(\mathrm{g})=\mathrm{g}$ o $\varnothing=\mathrm{g}(\mathrm{c})$ for $\mathrm{g} \in \mathrm{H}(\mathrm{Y})$ which implies $\mathbf{R}_{\varnothing}=$ $\Phi(\mathrm{H}(\mathrm{Y}))=\mathrm{C}$. Because of this contradiction $\varnothing$ is not a constant function.

Now we shall show that (b) implies (c). Suppose that $\varnothing$ is not a constant function and $\varnothing(\mathrm{X})$ is a non-empty subset of $\mathrm{Y}$. Let $\mathbf{f}$ and $\mathrm{g}$ be any two holomorphic functions on $\varnothing(X)$ belonging to $H(Y)$. Then there is an open set $\mathrm{U} \supset \varnothing(\mathrm{X})$ and functions $\mathrm{F}, \mathrm{G}$ holomorphic on $\mathrm{U}$ such that $f=F|\varnothing(X), g=G| \varnothing(X)$, respectively. Since $f-g$ is holomorphic on $\varnothing(\mathrm{X})$, it is clear that $\Phi(\mathrm{f})-\Phi(\mathrm{g})=\Phi(\mathrm{f}-\mathrm{g})=(\mathrm{f}-\mathrm{g})$ o $\varnothing$. Thus if $\Phi(\mathrm{f})-\Phi(\mathrm{g})=0$, then $\mathrm{f}-\mathrm{g}=0$ or equivalently $\mathrm{f}=\mathrm{g}$. This shows that $\Phi$ is an isomorphism.

Finally we shall show that (c) implies (a). If $\Phi$ is an isomorphism, then $\mathbf{R}_{\varnothing} \neq \mathrm{C}$ because $\mathrm{H}(\mathrm{Y})$ contains a non-constant function $\mathrm{g}$ [3] and if $\Phi(\mathrm{g})=\lambda$, a constant function, then the set $\Phi^{-1}(\lambda)$ would contain $\lambda$ and $g$ and $\Phi$ would not be one-to-one. Thus $R_{\varnothing}$ properly contains the constant function.

COROLLARY TO THEOREM 1. A subring $R^{*}$ of $H(X)$ is isomorphic to $H(Y)$ under a $C$-algebra isomorphism if and only if $R^{*}=$ $\{\mathrm{g} 0 \varnothing: \mathrm{g} \in \mathrm{H}(\mathrm{Y}), \varnothing \in \mathrm{A}(\mathrm{X}, \mathrm{Y})\}$ and $\mathrm{R}^{*}$ properly contains $\mathrm{G}$ the constant functions on $\mathrm{X}$.

In the following theorems we shall investigate some of the relations between $\mathbf{R}_{\varnothing}$ and $\varnothing$.

THEOREM 2. If $\varnothing$ is a one-to-one analytic mapping of $X$ on to $\mathrm{Y}$ and $\Phi$ maps $\mathrm{H}(\mathrm{Y})$ into $\mathrm{H}(\mathrm{X})$ by $\Phi(\mathrm{g})=\mathrm{g}$ o $\varnothing, \mathrm{g} \in \mathrm{H}(\mathrm{Y})$, then $\Phi$ $(\mathrm{H}(\mathrm{Y}))=\mathrm{H}(\mathrm{X})$. 
Proof. If $\varnothing$ is a one-to-one analytic mapping of $\mathrm{X}$ onto $\mathrm{Y}$, then $\varnothing^{-1}$ is a one-to-one function from $\mathrm{Y}$ onto $\mathrm{X}$. If $\mathrm{q}_{0} \in \mathrm{Y}$, then $\mathrm{p}_{0}=\varnothing^{-1}$ $\left(q_{0}\right) \in X$. By considering the definition of a Riemann surface and analiticity of a function between the non-empty subsets of two open Riemann surfaces we let $\mathrm{z}=\theta(\mathrm{p}), \mathrm{w}=\psi(\mathrm{g})$ be local parameters about $p_{0}$ and $q_{0}$ such that $\theta\left(p_{0}\right)=0, \psi\left(q_{0}\right)=0$. Then $\psi o \varnothing o \theta^{-1}(\mathrm{z})$ is analytic and one-to-one on $\left\{z:|z|<r_{1}\right\}$ for some $\mathbf{r}_{1}>0$ and 0 o $\varnothing^{-1}$ o $\psi^{-1}$ is analytic and one-to-one on $\left\{w:|w|<r_{2}\right\}$ for some $r_{2}>0$ since the inverse of a one-to-one analytic function is analytic. Thus $\varnothing^{-1}$ is a oneto-one analytic mapping of $\mathrm{Y}$ onto $\mathrm{X}$. If $\mathrm{f} \in \mathrm{H}(\mathrm{X})$, then $\mathrm{f}$ o $\varnothing^{-1} \in \mathbf{H}(\mathrm{Y})$ which implies (fo $\left.\varnothing^{-1}\right) \circ \varnothing=f \in \Phi(H(Y))$. This gives us $\Phi(H(Y))=$ $\mathrm{H}(\mathrm{X})$.

If $R_{\varnothing}=\Phi(H(Y))$ is to be a proper subring of $H(X)$, then $\varnothing$ may be one-to-one or onto or neither, but not both.

THEOREM 3. Suppose $\varnothing$ is a one-to-one analytic mapping of $X$ into $Y, \lambda$ is a non-constant analytic mapping of $X$ into $Y$ but not oneto-one, $\Phi(\mathrm{g})=\mathrm{g}$ o $\varnothing$ and $\Lambda(\mathrm{g})=\mathrm{g}$ o $\lambda$ for $\mathrm{g} \in \mathrm{H}(\mathrm{Y}), \mathbf{R}_{\varnothing}=\Phi(\mathrm{H}(\mathrm{Y}))$, $\mathbf{R}_{\lambda}=\Lambda(\mathbf{H}(\mathbf{Y}))$. Then $\mathbf{R}_{\varnothing}$ and $\mathbf{R}_{\lambda}$ are isomorphic but $\mathbf{R} \varnothing \neq \mathbf{R}_{\lambda}$.

Proof. $\Phi$ and $\Lambda$ are isomorphisms from $H(Y)$ onto $R_{\varnothing}$ and $R_{\lambda}$, respectively, so $\Lambda$ o $\Phi^{-1}$ is an isomorphism from $R_{\varnothing}$ onto $R_{\lambda}$. Suppose $\mathbf{R} \varnothing=\mathbf{R}_{\lambda}$. Let $\mathrm{g} \in \mathbf{H}(\mathbf{Y})$. Then there is $\mathbf{h} \in \mathbf{H}(\mathrm{Y})$ such that $\mathrm{g}$ o $\varnothing(\mathrm{z})=$ h o $\lambda(\mathrm{z}), \mathrm{z} \in \mathrm{X} . \varnothing$ is one-to-one and $\lambda$ is not one-to-one implies there are $z_{1}$ and $z_{2}$ in $X$ such that $z_{1} \neq z_{2}, \lambda\left(z_{1}\right)=\lambda\left(z_{2}\right)$ and $\varnothing\left(z_{1}\right) \neq \varnothing$ $\left(z_{2}\right)$. H (Y) separates the points of $Y[2]$ implies there is $g \in H(Y)$ such that $\mathrm{g}\left(\varnothing\left(\mathrm{z}_{1}\right)\right) \neq \mathrm{g}\left(\varnothing\left(\mathrm{z}_{2}\right)\right)$. But $\mathrm{g} \circ \varnothing\left(\mathrm{z}_{1}\right)=\mathrm{h}$ o $\lambda\left(\mathrm{z}_{1}\right)=\mathrm{h}$ o $\lambda\left(\mathrm{z}_{2}\right)=$ g o $\varnothing\left(\mathbf{z}_{2}\right)$. Since we reach a contradiction, $\mathbf{R}_{\varnothing} \neq \mathbf{R}_{\lambda}$.

\section{REFERENCES}

1. AHLFORS L. V. and SARIO L. Riemann surfaces, Princeton Math. Series no. 26 Princeton University Press Princeton, Fifth Printing, 1974.

2. BEHNKE, H., and SCHEJA, G., Studies in mathematical analysis and related topies, Stanford University Press 1962.

3. BEHNKE, H., and SOMMER, F., Theorie der analytischen Funktionen einar komplexen $V$ randerlichen, Springer-Verlag, Berlin, 1962.

4. MINDA C.D. Rings of holomorphic and meromorph'c functions on subsets of Riemann surfaces Journal of the Indian Math. Soc. 40 (1976) 75 85.

5. SPRINGER, G., Introduction to Riemann Surfaces, Addison-Wesley, Reading, Mass., 1967. 